\title{
Política social en Brasil y Cuba: un análisis crítico desde el subdesarrollo
}

\author{
Aline Fardin Pandolfi ${ }^{1}$ \\ https://orcid.org/0000-0002-3827-1792
}

\author{
Arelys Esquenazi Borrego ${ }^{2}$ \\ https://orcid.org/0000-0002-9366-8688
}

\author{
Camila Costa Valadão ${ }^{3}$ \\ https://orcid.org/0000-0001-9939-7308
}

${ }^{1}$ Universidade Federal do Espírito Santo, Departamento de Serviço Social, Vitória, ES, Brasil

${ }^{2}$ Universidade Federal do Espírito Santo, Programa de Pós-graduação em Política Social, Vitória, ES, Brasil

${ }^{3}$ Universidade Federal do Espírito Santo, Programa de Pós-graduação em Política Social, Vitória, ES, Brasil

\section{Política social en Brasil y Cuba: un análisis crítico desde el subdesarrollo}

Resumen: Este artículo tiene como objetivo analizar los fundamentos teóricos de la política social en Cuba y Brasil, a la luz de los proyectos de sociedad y perspectivas de desarrollo adoptadas por ambos países. La metodología utilizada es la revisión bibliográfica. El artículo está estructurado en tres secciones. Las dos primeras abordan los principales fundamentos teóricos de la política social en Cuba y Brasil. Posteriormente, los principales puntos de ruptura entre estas dos concepciones de política social se esbozan sintéticamente en las reflexiones finales. Se defiende la idea de que las manifestaciones, casi diametralmente opuestas, que asumen las políticas sociales en Cuba y Brasil están determinadas por sus fundamentos teóricos; que a su vez están determinados esencialmente por las distintas salidas que se adoptan para enfrentar el subdesarrollo en ambos países. Palabras clave: Política Social. Fundamentos teóricos. Subdesarrollo. Brasil. Cuba.

\section{Política social em Brasil e Cuba: uma análise crítica desde o subdesenvolvimento}

Resumo: Este artigo objetiva analisar os fundamentos teóricos da política social em Brasil e Cuba, à luz dos projetos de sociedade e perspectivas de desenvolvimento adotadas por esses países. A metodologia utilizada foi a revisão bibliográfica. $\mathrm{O}$ artigo está estruturado em três seções. As duas primeiras abordam os principais fundamentos teóricos da política social em Cuba e Brasil. Posteriormente, abordam-se os principais pontos de ruptura entre estas duas concepções de política social, onde se esboçam as reflexões finais. Evidencia-se que as manifestações, quase diametralmente opostas, que assumem as políticas sociais no Brasil e em Cuba estão determinadas pelos seus fundamentos teóricos, os que, por sua vez, estão essencialmente determinados pelas distintas saídas adotadas para enfrentar o subdesenvolvimento em ambos os países.

Palavras-chave: Política Social. Fundamentos teóricos. Subdesenvolvimento. Brasil. Cuba.

\section{Social policy in Brazil and Cuba: critical analysis from underdevelopment}

Abstract: This paper aims to analyze the theoretical foundations of social policy in Cuba and Brazil, in light of the societal projects and development perspectives adopted by both countries. The methodology used is the bibliographic review. The manuscript is structured in three sections. The first two address the main theoretical foundations of social policy for Cuba

(c) $\$$ Este es un artículo publicado en acceso (Open Access) abierto bajo la licencia Creative Commons Attribution BY NC Non-Commercial, que permite su uso, distribución y reproducción en cualquier medio, sin restricciones siempre que sin fines comerciales y que el trabajo original sea debidamente citado. 
and Brazil. Thereupon, the main points of rupture between these two conceptions of social policy are outlined synthetically. The article advocates that the almost diametrically opposed manifestations assumed by social policy in Brazil and Cuba are determined by their theoretical foundations; which in turn are essentially determined by the different solutions adopted to face underdevelopment in both countries.

Keywords: Social Policy. Theoretical foundations. Underdevelopment. Brazil. Cuba.

Recebido em 15.06.2020. Aprovado em 28.07.2020. Revisado em 15.10.2020.

\section{Introducción}

Cuba y Brasil son dos países de América Latina que presentan antecedentes históricos similares de colonización, exterminio indígena, esclavitud, plantaciones, etc. Posteriormente al período colonial ambos países avanzan en la institucionalización de una república formal, pero manteniendo una profunda relación de subordinación al capital internacional y Estados Unidos; presentando dicha subordinación particularidades en cada nación.

En este sentido, la condición de subdesarrollo ${ }^{1}$ históricamente determinada es otro elemento común a ambos países. Se trata de una situación de dependencia estructurante y sistémica, más allá de lo estrictamente económico; influyendo en otras esferas como lo social, político cultural, ambiental, entre otras.

Todos estos aspectos, se han expresado a lo largo del siglo XX en una serie de características socioeconómicas que, salvando algunas diferencias, también tienen puntos de coincidencia: estructuras económicas deformadas y heterogéneas; economías abiertas con un marcado carácter primario-exportador; elevado endeudamiento externo; creciente predominio y dominio del capital extranjero; latifundios; marcada desigualdad social; altas tasas de desempleo, analfabetismo, muertes por enfermedades prevenibles, etc.

Sin embargo, la salida de cada país planteada en el siglo pasado a estas condiciones históricas similares, fue realmente diferenciada para ambas naciones. Brasil y Cuba a partir de los años cincuenta adoptan, como respuesta a su condición dependencia y subdesarrollo, caminos opuestos en términos de proyectos societarios; proceso que a su vez implicó desdoblamientos diferenciados en las visiones de desarrollo. Las políticas sociales resultantes de este proceso comienzan a presentar enfoques, funciones y características muy diferentes entre ambos países.

Ello se hace explicito tanto en el papel que cumple la política social dentro de las respectivas visiones y estrategias de desarrollo esbozadas en cada uno de estos países; así como, en la forma concreta que asumen los diversos bienes y servicios asociados a las distintas esferas de la política social. Estas diferencias también son significativas en términos de acceso de la populación a dichos servicios, grado de cobertura, financiamiento, calidad, entre otros aspectos.

Se defiende la idea de que la manifestación concreta, casi diametralmente opuesta, que asumen las políticas sociales en cada uno de estos países, tanto en términos de concepción como de funcionamiento, guarda total relación a los fundamentos teóricos a partir de las cuales dichas políticas se estructuran. Este artículo tiene como objetivo analizar los fundamentos teóricos de la política social en Cuba y Brasil, a la luz de los proyectos societales y perspectivas de desarrollo asumidas por dichos países. La metodología utilizada es la revisión bibliográfica.

La elección de ambos países como objeto de análisis se encuentra determinada, fundamentalmente, por la necesidad de investigar, analizar críticamente y visibilizar otras alternativas diferentes a las que nos son colocadas para la política social como "únicas salidas posibles" dentro de la lógica del capital. Otra de las motivaciones está asociada al proyecto de investigación donde se encuentra insertado este estudio, el cual busca profundizar en términos de política social en estas dos experiencias latinoamericanas. ${ }^{2}$

El artículo, además de esta introducción, está estructurado en tres secciones. En las dos primeras se abordan, para Cuba y Brasil, los principales fundamentos teóricos de la política social; destacando sus implicaciones en términos de fortalezas y/o limitaciones. Posteriormente, se esbozan de forma sintética los principales puntos de ruptura entre estas dos concepciones de política social en las reflexiones finales. De esta forma, primero se aborda la experiencia de transición socialista cubana; destacando los avances que se producen en términos de construcción de una sociedad alternativa a partir de 1959 y sus desdoblamientos en la concepción de la 
política social cubana. Posteriormente, se analizan los fundamentos teóricos de la política social brasilera, en función de la perspectiva de desarrollo y de derechos ciudadanos refrendados en la Constitución de 1988; y sus posteriores desdoblamientos.

\section{Los fundamentos de la política social en Cuba: principales particularidades y desafíos.}

Cuba, dando continuidad a toda una historia de rebeldía, luchas independentistas y antiimperialistas que se remontan a 1868, emprende nuevamente en la segunda mitad de la década del cincuenta el camino de la Revolución. Su triunfo el 1ro de enero de 1959 asume una proyección democrática, popular, agraria, antiimperialista. Poco tiempo después, y debido a una serie de factores internos y externos, se produciría una profunda aceleración y radicalización del proceso revolucionario que se expresa en la declaración oficial en 1961 del carácter socialista de la Revolución por Fidel Castro; proceso que, al mismo tiempo, da inicio a una nueva etapa dentro de la historia de Cuba: el período de transición socialista.

A partir del triunfo revolucionario, y coherentemente con la intensión de cambio radical que a partir de este se gestó, fueron implementadas aceleradamente un conjunto de medidas de elevada repercusión socioeconómica, como respuesta a los problemas propios del subdesarrollo que caracterizaban al país en aquellos años. Entre las principales transformaciones impulsadas puede destacarse las siguientes: reforma agraria, reforma de la enseñanza, campaña de alfabetización, nacionalizaciones, reforma urbana, disminución de la tarifa eléctrica, garantía de alimentación básica subsidiada, desarrollo de servicios y programas sociales universales y gratuitos, etc. (Zanetti, 2013).

Todo ello representó la expresión concreta de un cambio de paradigma importante que va a ser consustancial al triunfo de la revolución cubana: "el surgimiento de una estrategia social como componente prioritario de un proyecto de nación independiente, regida por ideales de equidad y justicia en Cuba" (Espina, 2008, p. 135). En este sentido, el conjunto de programas, políticas, medidas, etc. implementadas en estos primeros años aunque no estuvieron denominadas explícitamente bajo la categoría de política social incluían, germinalmente, las bases de una estrategia de desarrollo social y de transformación estructural del patrón de desigualdades heredado (Voghon \& Peña, 2013).

Más allá de la ampliación de los derechos y garantías ciudadanas, estas medidas adicionalmente sustentaron una incipiente, pero sustancial, transformación de las relaciones de propiedad, producción y distribución; coherentes con los principios del imaginario de una sociedad alternativa. Es por ello que la formulación estratégica de la política social que se comienza a gestar en Cuba a partir de entonces se concreta en un enfoque que rebasa las tradicionales concepciones asistencialistas, compensatorias y residuales; para ubicarse en el terreno de la promoción del desarrollo (Odriozola et al., 2018) De esta forma, el papel activo de las políticas sociales y su relevancia dentro del proceso de desarrollo, se constituyó en una de las principales bases de la estrategia revolucionaria (Fuentes, 2013).

Es en este contexto donde Cuba también esboza e implementa una visión y práctica del desarrollo mucho más abarcadora. Donde lo económico y lo social no serían asumidos como aspectos separados; sino como dimensiones estrechamente articuladas, que se entrelazan y refuerzan mutuamente. Se trata del esbozo de una concepción más integradora del desarrollo, que abarcaría adicionalmente otras dimensiones relevantes como la: política, cultural, ambiental, tecnológica, entre otras (Espinosa, 2011; Pérez \& Esquenazi, 2018). Desde esta forma, el desarrollo es entendido como un proceso de carácter multicausal, multidimensional y sistémico.

Otro aspecto clave que ha distinguido al enfoque del desarrollo cubano es que se ha apropiado de la concepción humanista de la Revolución; colocando al sujeto social como centro del proceso de desarrollo (Pérez, 2018a). En el caso cubano, desde el comienzo, se explicita la necesidad ética del proceso de desarrollo en relación a los derechos humanos, la no discriminación, la equidad, la igualdad y la justicia social (Fuentes, 2013). En otras palabras, el ser humano es asumido como objeto y sujeto de la transformación social socialista (Pérez, 2018b).

Esta visión de desarrollo se ha intentado llevar a la práctica en las últimas seis décadas, a partir del diseño e implementación de disímiles estrategias de desarrollo, y su consecuente desdoblamiento en un conjunto de políticas económicas, sociales, jurídicas, institucionales, etc., que han ido adecuándose en función del contexto externo e interno. Cabe destacar que, aunque en el caso de la experiencia cubana la percepción 
acerca de un desarrollo integral estuvo presente desde el inicio del proceso revolucionario, ello no ha asegurado automáticamente, en la práctica, la relación adecuada entre todos los factores que tal desarrollo demandó (Fuentes, 2013).

Lo que sí resulta evidente cuando se analiza la concepción sobre el desarrollo y su implementación desde 1959 hasta hoy, es la relevancia concedida a la política social como uno de los principales elementos que distinguen al socialismo cubano; y en lo que quizás haya radicado una de sus principales fortalezas para enfrentar situaciones críticas de diversa naturaleza (Domínguez, 2006; Fuentes, 2013). Esto le ha permitido al país, a lo largo de más de seis décadas, alcanzar de forma sostenida importantes logros sociales en diversas esferas; los cuales han servido de referente tanto a países subdesarrollados como desarrollados.

Las principales esferas que integran la política social en Cuba son: educación, salud, cultura, deporte, seguridad y asistencia social, y satisfacción de necesidades nutricionales básicas (Espina, 2008; Pérez, 2011). Ello denota un enfoque más abarcador en lo relacionado tanto a las denominadas "necesidades básicas", como en las esferas del bienestar social; lo cual le ha permitido trascender las dimensiones tradicionales que asume la protección social dentro de los restrictos marcos de la lógica del capital.

En este sentido, es posible identificar como uno de sus fundamentos "una consideración del ser humano y de sus necesidades básicas amplia e integral, esto es, que no las restringe a la alimentación y a los servicios básicos, sino que incluye en estas relevantes elementos de la espiritualidad y la cultura" (Espina, 2012, p. 234), a partir de la comprensión de que la realización de las necesidades se va "desde el estómago a la fantasía" (Marx, 2008, p. 57). Las leyes, programas, medidas, acciones, etc. implementadas en las últimas seis décadas han tenido como principal objetivo la ampliación progresiva de los bienes y servicios asociados a estas siete áreas; lo cual se ha concretado en el desarrollo de una red de servicios públicos estable, abarcadora y de elevada calidad.

Todos estos elementos anteriormente destacados asociados a la visión cubana del desarrollo, han tenido un desdoblamiento en relación a los fundamentos teóricos de la propia política social. A continuación, se analizan críticamente algunos de estos aspectos, haciendo un balance de las principales fortalezas y desafíos del modelo de política social en Cuba a partir del examen de sus fundamentos teóricos.

En este sentido, el punto de partida es la identificación de la universalidad como principio básico y rector. Así, las características más relevantes de la política social cubana son: la cobertura total, la gratuidad o alto nivel de subsidio, y el carácter público de las políticas sociales y sus formas de realización. Por su parte, Espina (2017) destaca que una de las limitaciones que ha marcado a la politica social cubana es la identificación de universalidad con homogeneización de necesidades y satisfactores; lo cual por momentos ha conducido a una oferta universal de bienes y servicios extremadamente igualitaria. De ahí que, pese a las conquistas alcanzadas en términos de programas, acciones y resultados de indicadores sobre la base de la universalización; permanece latente el desafío de una mayor articulación entre políticas sociales universales dirigidas al conjunto de la población, y políticas sociales diferenciadas orientadas a grupos sociales específicos (Domínguez, 2006).

Asociado al corte universal, también tiene destaque su carácter centralmente planificado y unitario; donde el Estado es el máximo responsable en el diseño e implementación de la política social (Voghon \& Peña, 2013). Más allá del alto nivel de protagonismo del Estado como coordinador y gestor de la política social y el desarrollo, también constituye una característica fundamental el alto nivel de institucionalización de la política social. Ello se concreta en que ésta no sólo involucra a ministerios rectores y otras instancias de la administración pública, sino también a diferentes organizaciones políticas y sociales; así como, en el caso de algunas esferas específicas a la comunidad y las familias (Pérez, 2011).

Sin embargo, Espina (2012) destaca como ello ha implicado, en ocasiones, un diseño e implementación de las políticas sociales en la Isla marcadas en ciertos períodos de un excesivo centralismo y una elevada tecnoburocratización. De ahí que permanezcan retos tales como: ampliar el perfil de la participación popular a nivel de la formulación estratégica de la política social, consolidar un mayor balance entre centralización y descentralización en la implementación de las políticas sociales, potenciar una mayor complementación con los actores locales y no estatales, perfeccionar los procesos de seguimiento y evaluación de dichas políticas (Domínguez, 2006; Fundora, Hidalgo \& Díaz, 2017).

Dentro del conjunto abarcador de las políticas sociales en Cuba, pueden ser identificadas algunas áreas emblemáticas y priorizadas en la Isla. No obstante, ello también se presenta como una de las principales tensiones, dado que no siempre se ha producido un avance armónico y equilibrado entre las diferentes esferas 
que la componen (Espina, 2012). De esta forma, otro desafío que se perfila es el de avanzar hacia una mayor integralidad en el diseño e implementación de la política social, que supere la parcelación sectorial y los desbalances en términos de prioridades otorgadas a las diferentes esferas que la componen.

No obstante, cabe destacar que "a pesar de su condición de nación subdesarrollada, con limitados recursos naturales y fuertes tensiones financieras, Cuba se ha caracterizado por dedicar cuantiosos recursos a la esfera social" (Odriozola \& Colina, 2017, p. 202). De esta forma, el gasto de inversión social ha mantenido una tendencia creciente, con elevada estabilidad desde la década de los años sesenta hasta la actualidad, aún en los períodos de bajo crecimiento económico.

En este sentido, la política social cubana presenta desafíos específicos relacionados a la condición particular de un país en periodo de transición al socialismo. A su vez, el bloqueo económico, comercial y financiero que desde los primeros años de la Revolución fue impuesto por Estados Unidos también ha generado un conjunto de limitaciones de orden estructural que influyen en el bajo desarrollo de las fuerzas productivas. Todo ello impone toda una serie de desafíos adicionales para el proceso de desarrollo y la política social en Cuba.

En los últimos años, Cuba ha estado experimentando importantes transformaciones en las esferas económica, social y política, con base en el proceso de actualización del modelo económico y social. Formalmente iniciado en 2011 con la aprobación de los Lineamientos de Política Económica y Social del Partido y la Revolución, dicho proceso en 2016 amplía su base programática a partir de otros documentos como la Conceptualización del modelo y el Plan nacional de desarrollo económico y social hasta 2030.

Algunas de las transformaciones contenidas en dichos documentos prevén la preservación de los principios de universalidad, gratuidad e igualdad en áreas consideradas básicas, como educación, salud, cultura y deporte, que se proponen complementar con propuestas de políticas sociales direccionadas a distintos grupos vulnerables (Espina, 2017). De forma general, este proceso aunque ha significado una importante transformación de la política social sobre todo en su gestión; esta ha conservado lo mejor del núcleo duro de sus fundamentos teóricos (Odriozola et al. 2018). A la vez que ha introducido nuevos elementos de cara a superar algunas de las limitaciones que venían siendo identificadas (Fundora, Hidalgo \& Díaz, 2017).

\section{Austeridad y desmantelamiento de las políticas sociales en Brasil}

A lo largo de la década del cincuenta y principio de los años sesenta en Brasil, estaban apuntadas por la izquierda dos estrategias fundamentales. La primera seria la toma del poder político por los trabajadores y la desapropiación de los medios de producción de la élite nacional sin mediación con esta, en dirección a un proyecto de transición socialista. La segunda - una estrategia dominante a lo largo de la historia de luchas de clases en Brasil pasaría por una articulación con los representantes de la burguesía para desarrollar económicamente el país, a partir de la constitución de un proyecto democrático nacional y, después, iniciar una transición al socialismo. Esta segunda propuesta se constituyó en dos grandes ciclos, el primero se refiere al Partido Comunista Brasileño (PCB) con la estrategia nacional y democrática, el segundo ciclo se refiere al Partido de los Trabajadores (PT) con la estrategia democrático popular (Martins et al., 2019). Sin embargo, la estrategia de articulación de los movimientos o partidos de izquierda con la elite no se concretó en Brasil, y mucho menos las aspiraciones de una futura transición socialista a partir de ella.

En el gobierno de João Goulart se iniciaron cambios sociales importantes para la población a través de las Reformas de $\mathrm{Base}^{3}$, que rápidamente desagradaron al capital internacional. Como forma de impedir la aplicación de esta agenda de reformas de amplio alcance se articuló un golpe de estado en el año 1964 (Vieira, 1985). De esta forma, Brasil pasaría por un período de 20 años de Gobierno Militar, durante el cual se ejecutó radicalmente el proyecto del capital extranjero en el país. No obstante, al fuerte carácter represivo de la dictadura militar en dicho país, la lucha de clases se mantuvo desde la clandestinidad, a través de las organizaciones de grupos guerrilleros y las acciones lideradas por partidos políticos de izquierda, especialmente el PCB.

Con respecto a las políticas sociales en Brasil, hasta ese período, tuvieron una expansión lenta y selectiva, marcada por algunas pocas mejoras institucionales. El bloque militar-tecnocrático-empresarial dirigente de la dictadura buscó adhesión y legitimidad mediante la expansión y modernización de algunas políticas; al mismo tiempo que abrió espacios para el sector privado en la salud, la seguridad social y la educación, configurando un sistema de acceso doble (Behring \& Boschetti, 2007). 
La salida a este período de dictadura militar se produce en la segunda mitad de la década de los años ochenta y tiene como uno de sus momentos marcantes la aprobación de la Constitución de 1988. Ella consolida los derechos sociales, humanos y políticos en el marco de la forma republicana del Estado capitalista en la fase neoliberal (Behring \& Boschetti, 2007). En este contexto, fue implantado el Sistema Único de Salud (SUS) con una perspectiva universal, la Seguridad Social vía contribución, y la Asistencia Social para quien la necesitara.

Behring y Boschetti (2007) apuntan los múltiples determinantes de la política social. A partir de esa idea, las políticas sociales pasaron a ser implementadas en Brasil como resultantes de dos movimientos fundamentales. El primero, reproducir la fuerza de trabajo en función de un capitalismo dependiente; garantizando la expansión de la plusvalía en las formas relativa y absoluta. El segundo, como resultado de un largo proceso de lucha de clases en el país, antes y durante el período de dictadura militar.

En Brasil, la implementación de la agenda neoliberal se produce fundamentalmente a partir de los años noventa; comprobando una vez más el límite colocado a los avances de la política social y de mejoras en las condiciones de vida para los trabajadores en el interior del capitalismo dependiente brasileño. De esta forma, nunca se implementó la política social conforme previsto en la Carta Magna.

En coherencia con las pautas neoliberales implementadas en casi todas partes del mundo - siendo Cuba una de las escasas excepciones - el Brasil ha caminado hacia una tendencia cada vez más asistencialista de sus políticas sociales, dirigidas fundamentalmente a la población pobre y extremadamente pobre. De esta forma, la política social ha asumido un papel secundario y procíclico, en función de la expansión del capital ficticio a través de la liberalización financiera y la descompartimentalización de los mercados financieros (Marques, 2015).

En función de ello, una buena parte de las políticas sociales estructurantes como la salud, la educación y la seguridad social, se han mercantilizado, mientras que las políticas focales son ampliamente alentadas. A su vez, se produce un énfasis descentralizador caracterizado por el pasaje de funciones del Gobierno Federal para los demás entes federados, lo que ha generado una amplia responsabilización sobre todo financiera de los estados y municipios (Behring \& Boschetti, 2007). En resumen, la política social en Brasil que se comienza a desarrollar a partir de la década de los noventa, se consolida a partir de tres direccionamientos fundamentales: la privatización, la focalización y la descentralización.

Estos fundamentos de la política social brasilera se encontraban totalmente alineados con las tendencias mundiales. En el capitalismo contemporáneo, comandado por las finanzas, las políticas sociales basadas en el acceso universal son vistas no solo como poco "eficientes", sino que también imponen graves obstáculos para el cumplimiento de los requisitos de capital. Por ello, se observa una tendencia hacia la mercantilización de los derechos y la transmutación de las políticas sociales en servicios privados con el objetivo de ampliar las condiciones para la reproducción del capital a través de nuevos espacios de valorización (Granemann, 2007).

En esta línea, la política social llevada a cabo en Brasil en las últimas décadas, incluso en los gobiernos del Partido de los Trabajadores (PT) de 2002 hasta 2016 - que se encierra con el impeachment de Dilma Rousseff - aunque con algunas especificidades en este período, no implicó una ruptura esencial con las anteriormente apuntadas. Ejemplo de esto es que los gobiernos del PT no ampliaron de manera significativa las inversiones en políticas sociales; se mantuvo la Desvinculación de Recursos de la Unión (DRU) ${ }^{4}$; no realizaran la prometida Reforma Agraria; mantuvieron programas de transferencia de renta reuniéndolos en uno solo (que sigue hasta la actualidad) el "Programa Bolsa Familia"; realizaron varias alianzas público- privado que permitieron avanzar la enseñanza privada en el nivel superior, así como se promovieron algunos programas habitacionales a través de financiamiento bancario (Stocco, Pandolfi \& Nogueira, 2019). De esta forma, la política social se mantuvo apalancada en las propuestas del Banco Mundial y el Fondo Monetario Mundial, focalizadas en el enfrentamiento de la extrema pobreza.

En 2016, con el gobierno ilegítimo de Michel Temer, se aprueba la Emenda Constitucional 95 que congelan las inversiones del Gobierno Federal en las políticas sociales por 20 años (Brasil, 2016). Esto profundiza el proceso de precarización de los servicios públicos ofertados; al mismo tiempo que, desde el punto de vista ideológico, avanza la propaganda difamatoria de "lo público" como base del proyecto de privatización que se impulsa en el centro de la estructuración de las políticas sociales brasileñas. Se produce también la aprobación de la reforma de trabajo, expandiendo el desmonte de derechos sociales.

Electo en 2018 Jair Bolsonaro implementa una plataforma de acción basada en dos líneas, con tendencia al denominado protofascismo y ultraliberalismo. Ambos expresan lo que Fontes (2020) ha llamado de cabeza 
bifronte del Gobierno Bolsonaro. Esas son articuladas y funcionan conjuntamente con el objetivo único: la expansión del capitalismo en Brasil conforme el arreglo de la división internacional del trabajo, reforzando su carácter dependiente y más subordinado al capital internacional.

En ese sentido, en el Gobierno Bolsonaro fue aprobada la Reforma de las jubilaciones y pensiones, que constituye la más radical llevada a cabo desde la Constituición de 1998; alterando, entre otros aspectos, los parámetros que definen el valor y las condiciones de acceso a las-mismas (Stocco; Pandolfi; Nogueira, 2019). También se restringió el acceso al programa Bolsa Familia, disminuyeron las inversiones en las políticas de salud, asistencia social y educación, conforme EC 95 de 2016 (Brasil, 2016).

En resumen, se profundiza en los últimos años de forma acelerada el desmonte de los derechos sociales, la focalización, privatización, con vista a garantizar la reproducción del capital a través de nuevos espacios de valorización. Al mismo tiempo, se lleva al límite la función de la política social de reproducir la fuerza de trabajo. Así, la función de contención de la lucha de clases ha sido la función dominante de las políticas sociales en el capitalismo brasileño.

\section{Consideraciones finales}

El recorrido realizado a lo largo del presente artículo contribuye a argumentar la idea de que los fundamentos teóricos de las políticas sociales en Cuba y Brasil son coherentes con visiones de desarrollo y de proyectos de sociedad radicalmente opuestos, a partir de las salidas construidas por cada país a su condición de subdesarrollo. En este sentido la Tabla 1, que a continuación se presenta, sintetiza algunos de los principales elementos diferenciadores (de ruptura), en términos de los fundamentos teóricos de las políticas sociales, que distinguen a ambos modelos.

Tabla 1. Principales fundamentos teóricos de las políticas sociales en Cuba y Brasil.

\begin{tabular}{|c|c|}
\hline CUBA & BRASIL \\
\hline $\begin{array}{l}\S \text { Esencia humanista que se expresa en la visión de los sujetos } \\
\text { sociales como centro del proceso de desarrollo. }\end{array}$ & $\begin{array}{l}\text { § Resultante de la dualidad entre conquista y concesión; } \\
\text { mínima reproducción de la fuerza de trabajo y contención de la } \\
\text { lucha de clases. }\end{array}$ \\
\hline $\begin{array}{l}\text { § Permanencia y relevancia de las políticas sociales en las } \\
\text { estrategias de desarrollo a lo largo de los sesenta años de } \\
\text { Revolución. }\end{array}$ & $\begin{array}{l}\text { § Visión de desarrollo a partir de la lógica del capital: el } \\
\text { mercado como agente de las relaciones sociales. }\end{array}$ \\
\hline $\begin{array}{l}\text { § Centralidad de la igualdad, la equidad y la justicia social en } \\
\text { la formulación de las políticas sociales. }\end{array}$ & $\begin{array}{l}\text { § Límites de la igualdad y acceso a los derechos en la lógica } \\
\text { del capitalismo dependiente: neoliberalismo y pérdida de } \\
\text { derechos. }\end{array}$ \\
\hline $\begin{array}{l}\text { § Concepción de las políticas sociales basadas en la integración } \\
\text { social y la promoción del desarrollo individual, con un menor } \\
\text { peso del asistencialismo. }\end{array}$ & $\begin{array}{l}\text { § Retracción del carácter universal y expansión de la } \\
\text { focalización en la pobreza extrema, conforme recomendación } \\
\text { de los organismos internacionales. }\end{array}$ \\
\hline $\begin{array}{l}\S \text { Política social única, universal y centralizada. Planificación } \\
\text { centralizada como principal mecanismo para su ordenamiento. }\end{array}$ & $\begin{array}{l}\S \text { Orientación descentralizadora tanto en el diseño y como } \\
\text { en la implementación de las políticas sociales, acentuando } \\
\text { disparidades. regionales. }\end{array}$ \\
\hline$\S$ Elevado peso de la inversión social dentro del gasto público. & $\S$ Bajo peso de la inversión social dentro del gasto público. \\
\hline $\begin{array}{l}\S \text { Integración a partir del empleo. Protección del trabajador con } \\
\text { independencia de las formas de propiedad en la que se inserte. }\end{array}$ & $\begin{array}{l}\S \text { Pérdidas de derechos del trabajo, menor acceso a las políticas } \\
\text { sociales en general y con tendencia a su minimización. }\end{array}$ \\
\hline
\end{tabular}

Fuente: Elaboración propia.

Las perspectivas de desarrollo opuestas de estos países se expresan en los fundamentos teóricos opuestos que asumen sus políticas sociales. Diferencias que, en el contexto actual de pandemia por la COVID-19, se expresan aún más vívidamente. 
Más allá de las dificultades económicas de la condición de subdesarrollo y bloqueo económico que enfrenta Cuba, existe una política de salud totalmente pública, gratuita, estructurada centralmente, con un enfoque comunitario y de prevención. Por ello, para la enfrentar la pandemia Cuba cuenta con: instituciones de salud especializadas y con presencia en todo el país; personal de salud altamente calificados; tecnologías, equipamientos y medicamentos de producción nacional; elevada prioridad en términos de recursos y movilización de todos los actores sociales para salvaguardar la vida de las personas. Ello también se ha revertido en la cooperación internacional, con la presencia de equipos de médicos cubanos en varios países. En resumen, la visión de desarrollo y las políticas sociales en Cuba parten de un enfoque integral y de totalidad, dónde la economía busca responder a las necesidades sociales; lo cual se refleja en el actual combate a la pandemia.

En Brasil, por el contrario, las diferentes medidas adoptadas por el gobierno en el contexto de la pandemia han direccionado los esfuerzos a reactivar la economía, y fundamentalmente al gran capital, por encima de los recursos destinados a las políticas sociales. El discurso y acciones del gobierno ha priorizado a las ganancias por encima de la vida de las personas. Así, la población - que históricamente ha tenido retirados sus derechos sociales, ha enfrentado el lamentable dilema entre cumplir rigurosamente las reglas del distanciamiento social para prevenirse de la COVID-19 o arriesgarse a ir trabajar para intentar contener la situación de pobreza. En Brasil el Sistema Único de Salud (SUS) ya ha colapsado en varias ciudades y no ha podido, delante de tantos cortes de financiamiento y reducción de personal, mantener la capacidad de atención necesaria - que ya estaba tensionada en momentos de funcionamiento regular, menos aún en contexto actual de la pandemia.

Los días recientes han evidenciado una vez más, y de forma muy descarnada, a través de los efectos económicos y sociales de la pandemia, los límites y contradicciones de la sociedad capitalista. La actual crisis económica a nivel mundial que la pandemia profundiza, también evidencia la relevancia de pensar y construir proyectos de sociedad alternativos que - más allá de sus contradicciones y desafíos, como en el caso cubano - apuesten por un proyecto social y de desarrollo que se centre en las necesidades humanas.

\section{Referencias}

BEHRING, E. R., BOSCHETTI, I. (2007). Política Social: fundamentos e história. 2da. ed. São Paulo: Cortez.

BRASIL. (2016). [Constituição (1988)]. Emenda à Constituição n 55, de 2016. Altera o Ato das Disposições Constitucionais Transitórias, para instituir o Novo Regime Fiscal, e dá outras providências. Brasília, DF: Presidência da República. Recuperado de: https://www25.senado.leg.br/web/atividade/materias/-/materia/127337.

DOMÍNGUEZ, M. I. (2006). Políticas sociales y ciencias sociales en Cuba. Havana: CIPS.

ESPINA, M. (2008) Políticas de atención a la pobreza y la desigualdad: examinando el rol del Estado en la experiencia cubana. Buenos Aires: CLACSO. Recuperado de: http://biblioteca.clacso.edu.ar/clacso/clacso-crop/20100619071511/espina.pdf.

ESPINA, M. (2012). La política social en Cuba: nueva reforma económica. Revista de Ciencias Sociales, I-II(135-136), 227-236.

ESPINA, M. (2017). El bienestar como horizonte de políticas desde una teoría de las necesidades racionales. En: Zabala, M. C. Debates actuales sobre política social. Cuba en el contexto de América Latina y el Caribe. La Habana: FLACSO-Cuba, Fundación Friedrich Ebert. ESPINOSA, M. J. (2011). La Política Social y su rol en la transición socialista. Tesis en opción al grado de Máster en Economía. La Habana: Universidad de La Habana, Facultad de Economía.

FONTES, V. (2019). O protofascismo: arranjo institucional e policialização da existência. Revista Marxismo 21. Recuperado de: https://marxismo21.org/wp-content/uploads/2017/05/Virg\%C3\%ADnia-Fontes-O-protofascismo-\%E2\%80\%93-arranjo-institucionale-policializa\%C3\%A7\%C3\%A3o-da-exist\%C3\%AAncia.pdf.

FUENTES, S. (2013). Aproximación a las sinergias y tensiones entre "desarrollo social” y "desarrollo económico" en la Cuba revolucionaria. Un lugar desde el cual pensar el proceso de actualización del modelo cubano. Estudios del Desarrollo Social: Cuba y América Latina, 1(2), 54-66. Recuperado de: http://www.revflacso.uh.cu/index.php/EDS/article/view/19.

FUNDORA, G., HIDALGO, V., DÍAZ, D. (2017). Decidir la política social: concepciones, prácticas y proyecciones. En: Zabala, M. C. Debates actuales sobre política social. Cuba en el contexto de América Latina y el Caribe. La Habana: FLACSO-Cuba, Fundación Friedrich Ebert.

GRANEMANN, S. (2007). Políticas sociais e financeirização dos direitos do trabalho. Revista Em Pauta, (20), 56-68.

MARQUES, R. M. (2015). O lugar das políticas sociais no capitalismo contemporáneo. Revista Argumentum, Vitória, 7(2), 7-21.

MARTINS, C. et al. (2019). A "Estratégia Democrático-Popular” e um Inventário da Esquerda Revolucionária. En: Iasi, M. et al. A estratégia democrático popular: um inventário crítico. Marília: Lutas Anticapital. 
MARX. K. (2008). O Capital: Crítica da Economia Política. Rio de Janeiro: Civilização Brasileira. ODRIOZOLA, S. et al. (2018). Política Social: la experiencia de desarrollo cubano. En: Couto, B., Garcia, M. L., Pérez, O., Marques, R. Análisis dos sistemas de protección social na América Latina: Brasil, Chile e Cuba. Porto Alegre: EDIPUCRS.

ODRIOZOLA, S., COLINA, H. (2017). Política social y política económica en el contexto cubano actual. En: Zabala, M. C. Debates actuales sobre política social: Cuba en el contexto de América Latina y el Caribe. La Habana: FLACSO-Cuba, Fundación Friedrich Ebert. PÉREZ, O. (2011). La ciencia economica y los procesos de desarrollo: la experiencia cubana. Revista Argumentum, Havana, 3(2), 94-112. PÉREZ, O. (2018a). La política social es siempre economía y la economía es siempre economía política. Folleto 3. En: Pérez, O. De la ciencia económica que conocemos a la que necesitamos. La Habana: Editorial Universitaria Félix Varela.

PÉREZ, O. (2018b). El socialismo como ciencia económica del desarrollo. Lo necesario y posible. Folleto 5. En: Pérez, O. De la ciencia económica que conocemos a la que necesitamos. La Habana: Editorial Universitaria Félix Varela.

PÉREZ, O., ESQUENAZI, A. (2018). Protección y Política Social. En: Couto, B., Garcia, M. L., Pérez, O., Marques, R. Análisis dos sistemas de protección social na América Latina: Brasil, Chile e Cuba. Porto Alegre: EDIPUCRS.

STOCCO, A. F., PANDOLFI, A. F., NOGUEIRA, C.D.S. (2019). Golpe e Contrarreforma no Brasil: os limites do capitalismo dependente. Anais do Encontro Nacional e Internacional de Política Social, Espírito Santo, Vitória, 1(1).

VIEIRA, E. (1985). Estado e Miséria Social no Brasil de Getúlio a Geisel. 2da. ed. São Paulo: Cortez.

VOGHON, R. M., PEÑA, A. (2013). Reproducción de la pobreza en el contexto de políticas sociales universales? Encrucijadas y desafíos del modelo de protección social cubano. En: Robles, C. et al. Persistencias de la pobreza y esquemas de protección social en América Latina y el Caribe. Buenos Aires: CLACSO.

ZANETTI, O. (2013). Historia mínima de Cuba. España: Turner.

\title{
Notas
}

1 La condición de subdesarrollo es analizada a partir de las determinaciones que impone el proceso de acumulación capitalista y la división internacional del trabajo para dichos países. De esta forma, se suscribe la categoría subdesarrollo entendiéndola como una forma particular de desarrollo capitalista.

2 Este artículo se inserta dentro del proyecto de investigación "Alterações político-econômicas contemporâneas na América Latina e os impactos no seguro desemprego: o caso do Brasil e de Cuba". (Projecto FAPES: edital 22/2018, PPP)

3 En las Reformas de Base fueran propuestas: la Reforma Agraria mediante desapropiación de tierras; la Reforma Fiscal con el límite del envío de intereses al exterior; así como, un conjunto de reformas en el plano educacional, electoral, bancario y urbano.

4 Creada en 1994, permite que los Gobiernos utilicen 20\% de los recursos de la Unión para lo que consideren. En el Gobierno de Michel Temer (2017) ese valor pasó para 30\%. Los Gobiernos han utilizado ese recurso para el superávit primario.

\author{
Aline Fardin Pandolfi \\ ali_pandolfi@yahoo.com.br. \\ Doutora em Política Social pela Universidade Federal do Espírito Santo (UFES) \\ Professora do Departamento de Serviço Social da Universidade Federal do Espírito Santo (UFES)
}

\section{Arelys Esquenazi Borrego}

arelyseb@gmail.com

Doutoranda no Programa de Pós-graduação em Política Social da Universidade Federal do Espírito Santo (UFES)

\section{Camila Costa Valadão}

milacvaladao@gmail.com

Doutoranda no Programa de Pós-graduação em Política Social da Universidade Federal do Espírito Santo (UFES) 


\section{UFES}

Av. Fernando Ferrari, 514 - Goiabeiras

Vitória - Espirito Santo - Brasil

CEP: 29075-910

\section{Agradecimentos}

Agradecemos ao Programa de Pós-graduação em Política Social (PPGPS/ UFES) pelo profícuo espaço de formação acadêmica e pelas variadas formas de internacionalização. Agência financiadora

O presente trabalho foi realizado com apoio da Fundação de Amparo à Pesquisa e Inovação do Espírito Santo

(FAPES) através do projeto "Alterações político-econômicas contemporâneas na América Latina e os impactos no seguro desemprego: o caso do Brasil e de Cuba" (edital 22/2018, PPP) e da Coordenação de Aperfeiçoamento de Pessoal de Nível Superior (CAPES) - Código de financiamento 001.

\section{Contribuições das autoras}

Aline Fardin Pandolfi contribuiu com a concepção, redação e revisão crítica do artigo. Arelys Esquenazi Borrego contribuiu com a concepção, redação e revisão crítica do artigo. Camila Costa Valadão contribuiu com a redação e revisão crítica do artigo.Todas as autoras aprovaram a versão final do artigo. Aprovação por Comitê de Ética e consentimento para participação

Não aplica.

Consentimento para publicação

Consentimento das autoras.

Conflito de interesses

Não há conflito de interesses 